\title{
When is a matrix unitary or Hermitian plus low rank?*
}

\author{
Gianna M. Del Corso, Federico Poloni, Leonardo Robol, and \\ Raf Vandebril ${ }^{\dagger}$
}

November 20, 2018

\begin{abstract}
Hermitian and unitary matrices are two representatives of the class of normal matrices whose full eigenvalue decomposition can be stably computed in quadratic computing complexity. Recently, fast and reliable eigensolvers dealing with low rank perturbations of unitary and Hermitian matrices were proposed. These structured eigenvalue problems appear naturally when computing roots, via confederate linearizations, of polynomials expressed in, e.g., the monomial or Chebyshev basis. Often, however, it is not known beforehand whether or not a matrix can be written as the sum of an Hermitian or unitary matrix plus a low rank perturbation.

We propose necessary and sufficient conditions characterizing the class of Hermitian or unitary plus low rank matrices. The number of singular values deviating from 1 determines the rank of a perturbation to bring a matrix to unitary form. A similar condition holds for Hermitian matrices; the eigenvalues of the skew-Hermitian part differing from 0 dictate the rank of the perturbation. We prove that these relations are linked via the Cayley transform.

Based on these conditions we are able to identify the closest Hermitian and unitary plus low rank matrix in Frobenius and spectral norm and a practical Lanczos iteration to detect the low rank perturbation is presented. Numerical tests prove that this straightforward algorithm is robust with respect to noise.
\end{abstract}

\section{Introduction}

Normal matrices [26, 32, are computationally amongst the most pleasant matrices to work with. The fact that their eigenvectors form a full orthogonal set is the basic ingredient for developing many stable algorithms. Even though generic normal matrices are less common in practice the unitary and (skew-)Hermitian matrices are prominent members. Eigenvalue solvers for Hermitian [23, 24, 35] and unitary matrices [5, 15, 30, 31] have been examined thoroughly and well-tuned implementations are available in, e.g., eiscor [1] and LAPACK [2].

It has been noted that several linearizations of polynomials are rank one perturbations of unitary or Hermitian matrices [9,42, and a similar structure (with perturbations of higher

\footnotetext{
${ }^{*}$ The research of the first three authors was partially supported by GNCS projects "Metodi numerici avanzati per equazioni e funzioni di matrici con struttura" and "Tecniche innovative per problemi di algebra lineare"; the research of the first two was also supported by University of Pisa under the grant PRA-2017-05. The research of the fourth author was supported by the Research Council KU Leuven, project C14/16/056 (Inverse-free Rational Krylov Methods: Theory and Applications).

${ }^{\dagger}$ G. M. Del Corso at University of Pisa, Dept. Computer Science, Italy. gianna.delcorso@unipi.it and F. Poloni at University of Pisa, Dept. Computer Science, Italy. federico.poloni@unipi.it and L. Robol at Institute of Information Science and Technologies "A. Faedo", ISTI-CNR, Italy. leonardo.robol@isti.cnr.it and R. Vandebril at University of Leuven (KU Leuven), Dept. Computer Science, Belgium. raf.vandebril@cs.kuleuven.be.
} 
ranks) is found in the linearizations of matrix polynomials [11, 20, 21, 36 38. However, the development of eigenvalue solvers for matrices deviating only by a low rank perturbation from the unitary or Hermitian structure was much harder and solutions were proposed only recently. Most algorithms in this class rely on QR iterates 44] preserving the desired matrix structures, which are Hermitian, unitary, as well as the low rank structure. Retaining both these structures is numerically challenging, hence the various approaches and algorithms. Symmetric plus low rank solvers are encountered, for instance, when computing roots of polynomials expressed in bases that admit a three-terms recurrence such as the Chebyshev one. More generally, perturbations of low-rank structures arise when studying orthogonal polynomial on the real line, whereas unitary structures can be found in studying orthogonal polynomials on the unit circle [16, 42,

Algorithms to tackle the associated eigenvalue problems were developed by Chandrasekaran and $\mathrm{Gu}$ 18, Delvaux and Van Barel [22, Del Corso and Vandebril [41. One of the fastest and most stable versions is by Eidelman, Gemignani, and Gohberg [25. The unitary plus low rank eigenvalue problems received far more attention since the companion matrix, whose eigenvalues coincide with the roots of a polynomial in the monomial basis, fits in this class. Various algorithms differing with respect to storage-scheme, compression, explicit or implicit QR algorithms were proposed: Bini, Eidelman, Gemignani, and Gohberg [10]; Van Barel, Vandebril, Van Dooren, and Frederix 40; Chandrasekaran and Gu 19]; Boito, Eidelman, and Gemignani 13.14, Bevilacqua, Del Corso, Gemignani [7; and, a fast and provably stable version, is presented in the book of Aurentz, Mach, Robol, Vandebril, and Watkins [3. The general case of unitary plus low rank matrices has been recently addressed in [8]. Extensions for efficiently handling corrections with larger rank, necessary to deal with block companion matrices, have been recently presented in $[4,12,29]$.

In this article we will characterize unitary and Hermitian plus low rank matrices by examining their singular- and eigenvalues. Theorem 3 proves that a matrix having at most $k$ singular values less than 1 and at most $k$ singular values greater than 1 is of unitary plus rank $k$ form. Similarly, by examining the eigenvalues of the skew-Hermitian part of a matrix we show that at most $k$ eigenvalues greater than 0 and at most $k$ eigenvalues less than zero is compulsory for being of Hermitian plus rank $k$ form. The result is stated in Theorem 12. These analyses enable us to characterize the closest unitary or Hermitian plus rank $k$ matrices in the spectral and Frobenius norms by setting some well-chosen singular- or eigenvalues to 1 or 0 . We will also show that the Cayley transform bridges between the Hermitian and the unitary case. Up to our knowledge this is the first attempt of characterizing these low-rank perturbed matrices as well as solving the associated distance problems by considering the eigenvalues and singular-values. Finally, we have developed a numerical method based on the Lanczos iteration that allows us to recover the unitary/Hermitian and rank- $k$ parts explicitly in $\mathcal{O}\left(n^{2} k\right)$ flops.

The article is organized as follows. In Section 2 we revisit some preliminary results. Section 3 discusses the necessary and sufficient conditions for being of unitary plus low rank form. Constructive proofs furnish us the closest unitary plus low rank matrix in spectral and Frobenius norm. Sections 5 and 6 discuss similar topics, conditions for being of Hermitian plus low rank form and a manner of constructing the closest Hermitian plus low rank matrix. The Cayley transform, Section 7 allows us to transform the unitary into the Hermitian problem. We describe the Lanczos-based approach to compute the representation in Section 8 , and present some numerical experiments in Section 9. We conclude in Section 10. 


\section{Preliminaries}

In this text we make use of the following conventions. The symbols $I$ and 0 denote the identity and zero matrix, and may have subscripts denoting their size whenever that is not clear from the context. We use $\sigma_{1}(M) \geq \sigma_{2}(M) \geq \cdots \geq \sigma_{n}(M)$ to denote the singular values of a matrix $M \in \mathbb{C}^{n \times n}$, and $\lambda_{1}(H) \geq \lambda_{2}(H) \geq \cdots \geq \lambda_{n}(H)$ stand for the eigenvalues of a Hermitian matrix $H \in \mathbb{C}^{n \times n}$. We use the diag operator which stacks the objects, which could be scalars, matrices, or vectors, in a (pseudo-)diagonal matrix.

The following results are classical. We rely on them in the forthcoming proofs and for completeness we have included them.

Theorem 1 (Weyl's inequalities, 34, Theorem 4.3.16 and Exercise 16, page 423]). For every pair of matrices $M, N \in \mathbb{C}^{n \times n}$ and for every $i, j$ such that $i+j \leq n+1$,

$$
\sigma_{i+j-1}(M \pm N) \leq \sigma_{i}(M)+\sigma_{j}(N)
$$

If $M, N$ are Hermitian, then the same inequality holds for their eigenvalues.

$$
\lambda_{i+j-1}(M \pm N) \leq \lambda_{i}(M)+\lambda_{j}(N)
$$

Theorem 2 (Interlacing inequalities, [34, Theorems 4.3.4] and [39]). Let $M \in \mathbb{C}^{n \times n}$ and $N \in$ $\mathbb{C}^{n \times(n-k)}$ be a submatrix of $M$ obtained by removing $k$ columns from it. Then,

$$
\sigma_{i+k}(M) \leq \sigma_{i}(N) \leq \sigma_{i}(M)
$$

In the Hermitian case we get similar inequalities. Let $M \in \mathbb{C}^{n \times n}$ be Hermitian and $N \in$ $\mathbb{C}^{(n-k) \times(n-k)}$ be a (Hermitian) principal submatrix of $M$. Then,

$$
\lambda_{i+k}(M) \leq \lambda_{i}(N) \leq \lambda_{i}(M)
$$

Moreover, recall that $M \in \mathbb{C}^{n \times n}$ is unitary if and only if $\sigma_{i}(M)=1, \forall i=1, \ldots, n$.

\section{Detecting unitary-plus-rank- $k$ matrices}

We call $\mathcal{U}_{k}$ the set of unitary-plus-rank- $k$ matrices, i.e., $A \in \mathcal{U}_{k}$ if and only if there exists a unitary matrix $Q$ and two skinny matrices $G, B \in \mathbb{C}^{n \times k}$ such that $A=Q+G B^{*}$. This implies that $\mathcal{U}_{k} \subseteq \mathcal{U}_{k+1}$ for any $k$.

Theorem 3. Let $A \in \mathbb{C}^{n \times n}$ and $0 \leq k \leq n$. Then, $A \in \mathcal{U}_{k}$ if and only if $A$ has at most $k$ singular values strictly greater than 1 and at most $k$ singular values strictly smaller than 1 .

Before proving this result, we point out that looking at the singular values is a good guess, since being of unitary-plus-rank- $k$ form is invariant under unitary equivalence transformations.

Remark 4. $A=Q+G B^{*} \in \mathcal{U}_{k}$ if and only if $U^{*} A V \in \mathcal{U}_{k}$ for any unitary matrices $U, V$. Indeed, we have that

$$
U^{*} A V=\underbrace{U^{*} Q V}_{\hat{Q}}+\underbrace{U^{*} G}_{\hat{G}} \underbrace{B^{*} V}_{\hat{B}^{*}} \in \mathcal{U}_{k} .
$$

We start proving a simple case $(n=2, k=1)$ of Theorem 3 , which will act as a building block for the general proof. 
Lemma 5. For every pair of real numbers $\sigma_{1}$ and $\sigma_{2}$ such that $\sigma_{1} \geq 1 \geq \sigma_{2} \geq 0$, we have

$$
\left[\begin{array}{cc}
\sigma_{1} & 0 \\
0 & \sigma_{2}
\end{array}\right] \in \mathcal{U}_{1}
$$

Proof. We prove that the diagonal $2 \times 2$ matrix can be decomposed as a plane rotation plus a rank 1 correction. In particular, we look for $c, s, a, b \geq 0$ such that

$$
\left[\begin{array}{cc}
\sigma_{1} & 0 \\
0 & \sigma_{2}
\end{array}\right]=\left[\begin{array}{rr}
c & s \\
-s & c
\end{array}\right]+\left[\begin{array}{ll}
a & -s \\
s & -b
\end{array}\right]
$$

i.e., $\sigma_{1}=c+a$ and $\sigma_{2}=c-b$. In addition, we impose that the first summand is unitary (i.e., $c^{2}+s^{2}=1$ ), and that the second has rank 1 (i.e., $s^{2}=a b$ ). A simple computation shows that

$$
c=\frac{\sigma_{1} \sigma_{2}+1}{\sigma_{1}+\sigma_{2}}, \quad a=\frac{\sigma_{1}^{2}-1}{\sigma_{1}+\sigma_{2}}, \quad b=\frac{1-\sigma_{2}^{2}}{\sigma_{1}+\sigma_{2}}, \quad \text { and } s=\sqrt{a b}
$$

satisfy these conditions.

We are now ready to prove Theorem 3 .

Proof of Theorem 3. First note that the case $k=n$ is trivial $\mathcal{U}_{n}=\mathbb{C}^{n \times n}$. So we assume $k<n$. The conditions on the singular values can be written as two inequalities

$$
1 \geq \sigma_{k+1}(A) \text { and } \quad \sigma_{n-k}(A) \geq 1
$$

- We first show that if $A \in \mathcal{U}_{k}$ then the inequalities (1) hold. Suppose that $A=Q+G B^{*}$. Then, by Theorem 1 .

$$
\sigma_{k+1}(A) \leq \sigma_{1}(Q)+\sigma_{k+1}\left(G B^{*}\right)=1+0=1 .
$$

And, again following from Theorem 1 .

$$
1=\sigma_{n}(Q) \leq \sigma_{n-k}(A)+\sigma_{k+1}\left(G B^{*}\right)=\sigma_{n-k}(A) .
$$

- We now prove the reverse implication, i.e., if the two inequalities (11) hold then $A \in \mathcal{U}_{k}$. To simplify things, we introduce $k_{-}$denoting the number of singular values smaller than 1 and $k_{+}$standing for the number of singular values larger than 1 . The conditions state that $\ell=\max \left\{k_{-}, k_{+}\right\} \leq k$. We will prove that $A \in \mathcal{U}_{\ell}$. Note that $\mathcal{U}_{\ell} \subseteq \mathcal{U}_{k}$. Let $h=\min \left\{k_{-}, k_{+}\right\}$.

We reorder the diagonal elements of $\Sigma$ to group the singular values into separate diagonal blocks of three types:

- Diagonal blocks $\Sigma_{1}, \Sigma_{2}, \ldots, \Sigma_{h}$ of size $2 \times 2$, containing each one singular value larger than 1 and one smaller than 1 . Since $h=\min \left\{k_{-}, k_{+}\right\}$either all singular values smaller than 1 or all singular values larger than 1 are incorporated in these blocks.

- Diagonal blocks $\Sigma_{h+1}, \ldots, \Sigma_{\ell}$ of size $1 \times 1$ containing the remaining singular values different from 1 . Note that all these blocks will contain either singular values that are larger than 1 or smaller than 1 , depending on whether $h=k_{-}$or $h=k_{+}$. In case $k_{-}=k_{+}=h=\ell$ there will be no blocks of this type.

- One final block equal to the identity matrix of size $m=n-h-\ell=n-k_{-}-k_{+}$, containing all the singular values equal to 1 . 
For example,for $A$ having singular values $(3,2,2,1.3,1.2,1,1,1,0.6,0.2)$, we can take $\Sigma_{1}=$ $\operatorname{diag}(3,0.2), \Sigma_{2}=\operatorname{diag}(2,0.6), \Sigma_{3}=2, \Sigma_{4}=1.3, \Sigma_{5}=1.2$, and $\Sigma_{6}=I_{3}$.

Clearly, this decomposition always exists, and although it is not unique, the number and types of blocks are.

For each $i=1,2, \ldots, \ell$, the matrix $\Sigma_{i}$ is unitary plus rank 1 . This follows from Lemma 5 for $2 \times 2$ blocks, and is trivial for the $1 \times 1$ blocks. Hence for each $i$ we can write $\Sigma_{i}=Q_{i}+g_{i} b_{i}^{*}$, where $Q_{i}$ is unitary and $g_{i}, b_{i}$ are vectors. So we have

$$
\operatorname{diag}\left(\Sigma_{1}, \Sigma_{2}, \ldots, \Sigma_{\ell}, I_{m}\right)=\operatorname{diag}\left(Q_{1}, Q_{2}, \ldots, Q_{\ell}, I_{m}\right)+G B^{*}
$$

with

$$
G=\left[\begin{array}{c}
\operatorname{diag}\left(g_{1}, g_{2}, \ldots, g_{\ell}\right) \\
0_{m \times \ell}
\end{array}\right], \quad \text { and } \quad B=\left[\begin{array}{c}
\operatorname{diag}\left(b_{1}, b_{2}, \ldots, b_{\ell}\right) \\
0_{m \times \ell}
\end{array}\right] .
$$

Therefore $\operatorname{diag}\left(\Sigma_{1}, \Sigma_{2}, \ldots, \Sigma_{\ell}, I\right) \in \mathcal{U}_{\ell}$. Since this matrix can be obtained from a unitary equivalence on $A$ (singular value decomposition and a permutation) we have $A \in \mathcal{U}_{\ell}$.

Example 6. The matrix $U \operatorname{diag}(3,2,1,1,1,0.5) V^{*}$ belongs to $\mathcal{U}_{2}$ (but not to $\mathcal{U}_{1}$ ). The matrix $U \operatorname{diag}(5,0.4,0.3,0.2) V^{*}$ belongs to $\mathcal{U}_{3}$ (but not to $\mathcal{U}_{2}$ ). The matrix $5 I_{4}$ belongs to $\mathcal{U}_{4}$ (but not to $\left.\mathcal{U}_{3}\right)$.

\section{Distance from unitary plus rank $k$}

Theorem 3 provides an effective criterion to characterize matrices in $\mathcal{U}_{k}$ based on the singular values. One of the main features of the singular value decomposition is that it automatically provides the optimal rank $k$ approximation of any matrix, in the sense of the 2 -and the Frobenius norm. In this section, we show that the criterion of Theorem 3 can be used to compute the best unitary-plus-rank- $k$ approximant for any value of $k$.

The problem is thus to find a matrix in $\mathcal{U}_{k}$ that minimizes the distance to a given matrix $A$. This can be achieved by setting the supernumerary singular values preventing the inequalities (1) from being satisfied to 1 .

Theorem 7. Let the matrix $A \in \mathbb{C}^{n \times n}$ have singular value decomposition $U \Sigma V^{*}$. Then,

$$
\arg \min _{X \in \mathcal{U}_{k}}\|A-X\|_{2}=\hat{A}:=U \widehat{\Sigma} V^{*}
$$

where $\widehat{\Sigma}$ is the diagonal matrix with diagonal elements

$$
\hat{\sigma}_{i}= \begin{cases}1 & \text { if } k<i \leq k_{+}, \text {or } n-k_{-}<i \leq n-k, \\ \sigma_{i} & \text { otherwise }\end{cases}
$$

with $k_{+}$(resp. $\left.k_{-}\right)$the number of singular values of $A$ strictly greater (resp. smaller) than 1. Moreover, we have $\|A-\widehat{A}\|_{2}=\max \left\{\sigma_{k+1}-1,1-\sigma_{n-k}\right\}$.

Proof. The matrix $\hat{A}=U \widehat{\Sigma} V^{*}$ clearly belongs to $\mathcal{U}_{k}$ by Theorem [3] hence $\left\|A-U \widehat{\Sigma} V^{*}\right\|_{2}=$ $\|\Sigma-\widehat{\Sigma}\|_{2}=\max \left\{\sigma_{k+1}-1,1-\sigma_{n-k}, 0\right\}$. It remains thus to prove that for every $X \in \mathcal{U}_{k}$ one has $\|A-X\|_{2} \geq\|A-\hat{A}\|_{2}$. 
By Weyl's inequality (Theorem 1), we have that $\sigma_{k+1}(A) \leq \sigma_{1}(A-X)+\sigma_{k+1}(X)$, and therefore

$$
\|A-X\|_{2} \geq \sigma_{k+1}(A)-\sigma_{k+1}(X) \geq \sigma_{k+1}(A)-1 .
$$

Similarly from $\sigma_{n-k}(X) \leq \sigma_{1}(A-X)+\sigma_{n-k}(A)$, we have

$$
\|A-X\|_{2} \geq \sigma_{n-k}(X)-\sigma_{n-k}(A) \geq 1-\sigma_{n-k}(A) .
$$

We obtain

$$
\|A-X\|_{2} \geq \max \left\{\sigma_{k+1}-1,1-\sigma_{n-k}, 0\right\}=\|A-\hat{A}\|_{2} .
$$

Theorem 7 provides a deterministic construction for a minimizer of $\|A-X\|_{2}$, but this minimizer is not unique. This is demonstrated in the following example.

Example 8. Let us consider, for arbitrary unitary matrices $U, V$, the matrix $A$ defined as

$$
A=U\left[\begin{array}{lllll}
2 & & & & \\
& 1.5 & & & \\
& & 1 & & \\
& & & 1 & \\
& & & & .5
\end{array}\right] V^{*}
$$

We know, from Theorem 3 that $A \in \mathcal{U}_{2}$. We want to determine the distance from $A$ to $\mathcal{U}_{1}$. Theorem 7 yields the approximant $\hat{A}$ defined as follows:

$$
\hat{A}:=U\left[\begin{array}{ccccc}
2 & & & & \\
& 1 & & & \\
& & 1 & & \\
& & & 1 & \\
& & & & .5
\end{array}\right] V^{*}, \quad\|A-\hat{A}\|_{2}=0.5 .
$$

However, the solution is not unique. For instance, the family of matrices determined as

$$
\hat{A}(t, s):=U\left[\begin{array}{ccccc}
2+t & & & & \\
& 1 & & & \\
& & 1 & & \\
& & & 1 & \\
& & & & .5+s
\end{array}\right] V^{*}
$$

still lead to $\|A-\hat{A}(t, s)\|_{2}=0.5$ for $t, s \in[-0.5,0.5]$.

The same matrix is a minimizer also in the Frobenius norm.

Theorem 9. Let the matrix $A \in \mathbb{C}^{n \times n}$ have singular value decomposition $U \Sigma V^{*}$. Then,

$$
\arg \min _{X \in \mathcal{U}_{k}}\|A-X\|_{F}=\hat{A}:=U \widehat{\Sigma} V^{*},
$$

where $\widehat{\Sigma}$ is the diagonal matrix with diagonal elements

$$
\hat{\sigma}_{i}= \begin{cases}1 & \text { if } k<i \leq k_{+}, \text {or } n-k_{-}<i \leq n-k, \\ \sigma_{i} & \text { otherwise }\end{cases}
$$


where $k_{+}\left(\right.$resp. $\left.k_{-}\right)$is the number of singular values of $A$ which are strictly greater (resp. smaller) than 1 . Moreover we have

$$
\|A-\hat{A}\|_{F}^{2}=\sum_{i=k+1}^{k_{+}}\left(\sigma_{i}-1\right)^{2}+\sum_{i=n-k_{-}+1}^{n-k}\left(\sigma_{i}-1\right)^{2} .
$$

Proof. Since the Frobenius norm is unitarily invariant, we immediately have (2). To complete the proof, we show that for an arbitrary $X \in \mathcal{U}_{k}$ we have $\|A-X\|_{F} \geq\|A-\hat{A}\|_{F}$. Let $\Delta$ be the matrix such that $X=A+\Delta \in \mathcal{U}_{k}$. We will prove that $\|\Delta\|_{F}^{2} \geq\|A-\hat{A}\|_{F}^{2}$. Consider $\tilde{\Delta}=U^{*} \Delta V$ and partition

$$
U^{*} X V=U^{*}(A+\Delta) V=\left[\begin{array}{llll}
\Sigma_{1}+\tilde{\Delta}_{1} & \Sigma_{2}+\tilde{\Delta}_{2} & \Sigma_{3}+\tilde{\Delta}_{3}
\end{array}\right]
$$

where $\Sigma_{1} \in \mathbb{C}^{n \times k_{+}}$contains the singular values of $A$ which are larger than $1, \Sigma_{2}$ the singular values equal to 1 , and $\Sigma_{3} \in \mathbb{C}^{n \times k_{-}}$the singular values smaller than 1 .

Since $U^{*}(A+\Delta) V \in \mathcal{U}_{k}$, we have

$$
\sigma_{k+1}\left(U^{*}(A+\Delta) V\right) \leq 1, \quad \sigma_{n-k}\left(U^{*}(A+\Delta) V\right) \geq 1 .
$$

- Assume first that $k_{+}>k$. By the interlacing inequalities (Theorem 2),

$$
\sigma_{k+1}\left(\Sigma_{1}+\tilde{\Delta}_{1}\right) \leq \sigma_{k+1}\left(U^{*}(A+\Delta) V\right) \leq 1,
$$

and then by Weyl's inequalities (Theorem 1) for each $k<i \leq k_{+}$

$$
\sigma_{i}=\sigma_{i}\left(\Sigma_{1}\right) \leq \sigma_{k+1}\left(\Sigma_{1}+\tilde{\Delta}_{1}\right)+\sigma_{i-k}\left(\tilde{\Delta}_{1}\right) \leq 1+\sigma_{i-k}\left(\tilde{\Delta}_{1}\right),
$$

from which we obtain $\sigma_{i-k}\left(\tilde{\Delta}_{1}\right) \geq \sigma_{i}-1$, and hence

$$
\left\|\tilde{\Delta}_{1}\right\|_{F}^{2} \geq \sum_{i=1}^{k_{+}-k} \sigma_{i}\left(\tilde{\Delta}_{1}\right)^{2} \geq \sum_{i=k+1}^{k_{+}}\left(\sigma_{i}-1\right)^{2} .
$$

Note that (3) holds trivially also when $k_{+} \leq k$.

- Similarly, assume that $k_{-}>k$ (the case $k_{-} \leq k$ is trivial), and we use interlacing inequalities (Theorem 2) to get

$$
\sigma_{k_{--k}}\left(\Sigma_{3}+\tilde{\Delta}_{3}\right) \geq \sigma_{n-k}\left(U^{*}(A+\Delta) V\right) \geq 1,
$$

and Weyl's inequalities (Theorem 11) for each $k<i \leq k_{-}$to show

$$
1 \leq \sigma_{k_{-}-k}\left(\Sigma_{3}+\tilde{\Delta}_{3}\right) \leq \sigma_{k_{-}+1-i}\left(\Sigma_{3}\right)+\sigma_{i-k}\left(\tilde{\Delta}_{3}\right)=\sigma_{n+1-i}+\sigma_{i-k}\left(\tilde{\Delta}_{3}\right),
$$

from which we obtain $\sigma_{i-k}\left(\tilde{\Delta}_{3}\right) \geq 1-\sigma_{n+1-i}$. Hence

$$
\left\|\tilde{\Delta}_{3}\right\|_{F}^{2} \geq \sum_{j=n+1-k_{-}}^{n-k}\left(1-\sigma_{j}\right)^{2} .
$$

Putting together (3) and (4), we have

$$
\|\Delta\|_{F}^{2} \geq\left\|\tilde{\Delta}_{1}\right\|_{F}^{2}+\left\|\tilde{\Delta}_{3}\right\|_{F}^{2} \geq \sum_{i=k+1}^{k_{+}}\left(\sigma_{i}-1\right)^{2}+\sum_{j=n+1-k_{-}}^{n-k}\left(1-\sigma_{j}\right)^{2}=\|A-\hat{A}\|_{F}^{2},
$$

which is precisely what we wanted to prove. 
In this case, unlike in the 2-norm setting, this minimizer is unique when all singular values are different 1

\section{Detecting Hermitian-plus-rank- $k$ matrices}

We call $\mathcal{H}_{k}$ the set of Hermitian-plus-rank- $k$ matrices, i.e., $A \in \mathcal{H}_{k}$ if and only if there exist a Hermitian matrix $H$ and two matrices $G, B \in \mathbb{C}^{n \times k}$ such that $A=H+G B^{*}$.

In this and the next section we will answer similar questions: how can we tell if $A \in \mathcal{H}_{k}$ ? How do we find the distance from a matrix $A$ to the closed set $\mathcal{H}_{k}$ ? To answer these questions, we first need an Hermitian equivalent of Lemma 5 .

Lemma 10. For any pair of real numbers $\sigma_{1}$ and $\sigma_{2}$, such that $\sigma_{1} \geq 0, \sigma_{2} \leq 0$, there are two real vectors $c$ and $b$ such that

$$
\left[\begin{array}{ll}
\sigma_{1} & \\
& \sigma_{2}
\end{array}\right]=b c^{T}+c b^{T} .
$$

Proof. Define the vectors $c$ and $b$ as follows:

$$
b:=\frac{1}{2}\left[\begin{array}{c}
\sqrt{\sigma_{1}} \\
-\sqrt{-\sigma_{2}}
\end{array}\right], \quad c:=\left[\begin{array}{c}
\sqrt{\sigma_{1}} \\
\sqrt{-\sigma_{2}}
\end{array}\right] .
$$

The result follows by a direct computation.

The next Theorem is the analogue of Theorem 3, where we look at eigenvalues of the skewHermitian part of a matrix instead of at the singular values. We rely on the following lemma.

Lemma 11. Let $B, C$ be any $n \times k$ full rank matrices, and let $S=B C^{*}+C B^{*}$. Then, $S$ has at most $k$ positive and at most $k$ negative eigenvalues.

Proof. Up to a change of basis, we can assume $C=\left[\begin{array}{c}I_{k} \\ 0\end{array}\right]$. Then, $S$ has a trailing $(n-k) \times$ $(n-k)$ zero submatrix $T$. Let $\lambda_{1}(S) \geq \cdots \geq \lambda_{n}(S)$ be the eigenvalues of $S$. By the interlacing inequalities, $\lambda_{k+1}(S) \leq \lambda_{1}(T)=0$, and $\lambda_{n-k}(S) \geq \lambda_{n-k}(T)=0$.

Theorem 12. Let $A \in \mathbb{C}^{n \times n}$, and $0 \leq k \leq n$. Then, $A \in \mathcal{H}_{k}$ if and only if the Hermitian matrix $S(A):=\frac{1}{2 i}\left(A-A^{*}\right)$ has at most $k$ positive eigenvalues and at most $k$ negative eigenvalues.

Proof. Let $\lambda_{1}, \ldots, \lambda_{n}$ be the eigenvalues of $\frac{1}{2 i}\left(A-A^{*}\right)$, sorted by decreasing order, i.e., $\lambda_{1} \geq$ $\lambda_{2} \geq \cdots \geq \lambda_{n}$. Then the stated condition is equivalent to demanding $\lambda_{k+1} \leq 0, \lambda_{n-k} \geq 0$.

We first show that if $A \in \mathcal{H}_{k}$ these inequalities hold. If $A \in \mathcal{H}_{k}$, then there exists a Hermitian matrix $H$ and two matrices $G, B \in \mathbb{C}^{n \times k}$ such that $A=H+G B^{*}$. Then

$$
\frac{1}{2 i}\left(A-A^{*}\right)=\frac{1}{2 i}\left(G B^{*}-B G^{*}\right)=C B^{*}+B C^{*},
$$

with $C=G /(2 i)$. The result follows from Lemma 11]

We now prove the converse, that is, each matrix satisfying the inequalities belongs to $\mathcal{H}_{k}$. We first prove that each Hermitian matrix $S$ that has $k_{+}$strict positive eigenvalues and $k_{-}$ strict negative eigenvalues, where $\ell=\max \left\{k_{-}, k_{+}\right\} \leq k$ can be written as $C B^{*}+B C^{*}$ with $B, C \in \mathbb{C}^{n \times k}$. Let us assume that the eigenvalues of $S$ are $\lambda_{j}$ with

$$
\lambda_{1}, \ldots, \lambda_{k_{+}}>0, \quad \lambda_{k_{+}+1}, \ldots, \lambda_{k_{+}+k_{-}}<0, \quad \lambda_{k_{+}+k_{-}+1}, \ldots, \lambda_{n}=0 .
$$

\footnotetext{
${ }^{1}$ In fact the constraint of all singular values differing can be relaxed: one can construct examples in which there are several identical singular values and all (or none) of them should be changed to 1 .
} 
Since $S$ is normal, we can diagonalize it by an orthogonal transformation and obtain

$$
Q^{*} S Q=\operatorname{diag}\left(\Lambda_{1}, \ldots, \Lambda_{h}, \Lambda_{h+1}, \ldots, \Lambda_{\ell}, 0_{m}\right),
$$

where we get, similar as in the proof of the unitary case, three types of blocks:

- The diagonal blocks $\Lambda_{1}, \Lambda_{2}, \ldots, \Lambda_{h}$ of size $2 \times 2$ containing one eigenvalue larger and one eigenvalue smaller than 0 .

- The $1 \times 1$ matrices $\Lambda_{h+1}, \ldots, \Lambda_{\ell}$ containing the remaining eigenvalues differing from 0 . Since either all positive or negative eigenvalues are used already in the blocks $\Lambda_{1}$ up to $\Lambda_{h}$ we end up with scalar blocks of all the same sign.

- A final zero matrix of size $m=n-k_{-} k_{+}=n-h-\ell$.

Lemma 10 tells us that each of the $2 \times 2$ blocks $\Lambda_{j}$, for $j=1, \ldots, h$ can be written as $b_{j} c_{j}^{*}+c_{j} b_{j}^{*}$, for appropriate choices of $b_{j}, g_{j}$, since the eigenvalues on the diagonal are real and have opposite sign. Moreover, the remaining diagonal entries $\Lambda_{j}$ for $j=h+1, \ldots, \ell$ can be written choosing $b_{j}=\lambda_{j}$ and $g_{j}=1 / 2$. Therefore, we conclude that $Q^{*} S Q$ is of the form $\tilde{C} \tilde{B}^{T}+\tilde{B} \tilde{C}^{T}$ for some $\tilde{C}, \tilde{B}$ with $k_{-}$columns. Setting $G:=(-2 i) Q \tilde{C}$ and $B:=Q \tilde{B}$ proves our claim. It is immediate to verify that the matrix $A-G B^{*}$ is Hermitian, since $\left(A-G B^{*}\right)^{*}-\left(A-G B^{*}\right)=0$.

Theorem 12 has alternative formulations as well. We can for instance look at $A-A^{*}$ and count the number of eigenvalues with positive and negative imaginary parts, since when $A$ is Hermitian, $i A$ will be skew-Hermitian.

We will not go into the details, but it is obvious that Theorem 12 admits an equivalent formulation to check whether a matrix is a rank $k$ perturbation of a skew-Hermitian matrix. To this end one considers $A+A^{*}$ and counts the number of positive and negative eigenvalues.

\section{Distance from Hermitian plus rank $k$}

In this section we will construct, given an arbitrary matrix $A$, the closest Hermitian-plus-rank- $k$ matrix in both the 2- and the Frobenius norm. The following lemma comes in handy.

Lemma 13. Let $\|\cdot\|$ be any unitarily invariant norm, and $X \in \mathbb{C}^{n \times n}$, and $S(X)=\frac{X-X^{*}}{2 i}$. Then, $\|S(X)\| \leq\|X\|$.

Proof. Let $X=U \Sigma V^{*}$ be the SVD of $X$. Since $\|\cdot\|$ is unitarily invariant, we have that $\left\|X^{*}\right\|=$ $\left\|V \Sigma^{*} U^{*}\right\|=\|\Sigma\|=\left\|U \Sigma V^{*}\right\|=\|X\|$. Then,

$$
\left\|\frac{X-X^{*}}{2 i}\right\| \leq \frac{\|X\|+\left\|X^{*}\right\|}{2}=\|X\| .
$$

We formulate again two Theorems, one for the closest approximation in the 2-norm and another one for the closest approximation in the Frobenius norm. The approximants that we construct are identical, but the proofs differ significantly. Again like in the unitary case we will change particular eigenvalues of the skew-Hermitian part to find the best approximant.

Theorem 14. Let $A \in \mathbb{C}^{n \times n}$ and $S(A)=\frac{1}{2 i}\left(A-A^{*}\right)$, having eigendecomposition $S(A)=Q D Q^{*}$. The eigenvalues are ordered: $\lambda_{1} \geq \cdots \geq \lambda_{k_{+}}>0, \lambda_{k_{+}+1}=\cdots=\lambda_{n-k_{-}}=0$ and $0>\lambda_{n-k_{-}+1} \geq$ $\cdots \geq \lambda_{n}$, where $k_{+}$stands for the number of eigenvalues strictly greater than 0 and $k_{-}$for the eigenvalues strictly smaller than 0 . Then we have that

$$
\arg \min _{X \in \mathcal{H}_{k}}\|A-X\|_{2}=\hat{A}:=A-i Q(D-\hat{D}) Q^{*},
$$


where $\hat{D}$ is a diagonal matrix with diagonal elements

$$
\hat{d}_{i}= \begin{cases}0 & \text { if } k<i \leq k_{+} \text {or } n-k_{-}<i \leq n-k \\ \lambda_{i} \quad \text { otherwise. } & \end{cases}
$$

Proof. Theorem 12 implies that $\hat{A}=A-i Q(D-\hat{D}) Q^{*}$ belongs to $\mathcal{H}_{k}$, since

$$
S(\hat{A})=\frac{\hat{A}-\hat{A}^{*}}{2 i}=\frac{A-A^{*}}{2 i}-Q(D-\hat{D}) Q^{*}=Q \hat{D} Q^{*},
$$

has at most $k$ positive eigenvalues and at most $k$ negative eigenvalues.

To prove that $\hat{A}$ is the minimizer of $\|A-X\|_{2}$ we have to prove that for every $X \in \mathcal{H}_{k}$ we have that $\|A-X\|_{2} \geq\|A-\hat{A}\|_{2}=\|D-\hat{D}\|_{2}=\max \left\{\lambda_{k+1}(S(A)),-\lambda_{n-k}(S(A))\right\}$.

Assume that $X=A+\Delta$, then $S(X)=S(A)+S(\Delta)$. Using Weyl's inequality (Theorem 1), we have

$$
\lambda_{k+1}(S(X))=\lambda_{k+1}(S(A)+S(\Delta)) \geq \lambda_{k+1}(S(A))-\lambda_{1}(S(\Delta))
$$

and therefore

$$
\|A-X\|_{2}=\|\Delta\|_{2} \geq\|S(\Delta)\|_{2}=\lambda_{1}(S(\Delta)) \geq \lambda_{k+1}(S(A))-\lambda_{k+1}(S(X)) \geq \lambda_{k+1}(S(A))
$$

since $X \in \mathcal{H}_{k}$ implies $\lambda_{k+1}(S(X)) \leq 0$. Similarly from

$$
\lambda_{n-k}(S(A+\Delta)) \leq \lambda_{1}(S(\Delta))+\lambda_{n-k}(S(A)),
$$

we get

$$
\|A-X\|_{2} \geq \lambda_{1}(S(\Delta)) \geq \lambda_{n-k}(S(X))-\lambda_{n-k}(S(A)) \geq-\lambda_{n-k}(S(A)),
$$

since $X \in \mathcal{H}_{k}$ implies $\lambda_{n-k}(S(X)) \geq 0$. Combining the two inequalities and using the nonnegativeness of the norm, we have

$$
\|A-X\|_{2} \geq \max \left\{\lambda_{k+1}(S(A)),-\lambda_{n-k}(S(A))\right\} .
$$

We remark that, comparable to the unitary case, the minimizer in the 2-norm is not unique. We have constructed a solution $\hat{A}$ such that $\|A-\hat{A}\|_{2}=\|D-\hat{D}\|_{2}=\max \left\{\lambda_{k+1}(S(A)),-\lambda_{n-k}(S(A))\right\}$. It is, however, easy to find a concrete example and a matrix $\tilde{A}$ different from $\hat{A}$ such that $\|A-\hat{A}\|_{2}=\|D-\hat{D}\|_{2}=\|D-\tilde{D}\|_{2}=\|A-\tilde{A}\|_{2}$.

Theorem 15. Let $A \in \mathbb{C}^{n \times n}$ and $S(A)=\frac{1}{2 i}\left(A-A^{*}\right)$, having eigendecomposition $S(A)=Q D Q^{*}$. The eigenvalues are ordered: $\lambda_{1} \geq \cdots \geq \lambda_{k_{+}}>0, \lambda_{k_{+}+1}=\cdots=\lambda_{n_{-} k_{-}}=0$ and $0>\lambda_{n-k_{-}+1} \geq$ $\cdots \geq \lambda_{n}$, where $k_{+}$stands for the number of eigenvalues strictly greater than 0 and $k_{-}$for the eigenvalues strictly smaller than 0 . Then we have that

$$
\arg \min _{X \in \mathcal{H}_{k}}\|A-X\|_{F}=\hat{A}:=A-i Q(D-\hat{D}) Q^{*},
$$

where $\hat{D}$ is a diagonal matrix with diagonal elements

$$
\hat{d}_{i}= \begin{cases}0 & \text { if } k<i \leq k_{+} \text {or } n-k_{-}<i \leq n-k \\ \lambda_{i} \quad \text { otherwise. }\end{cases}
$$


Proof. We know that $\hat{A} \in \mathcal{H}_{k}$. To prove that this is the minimizer, we have to show that for any $X \in \mathcal{H}_{k}$ we have that $\|A-X\|_{F} \geq\|A-\hat{A}\|_{F}$.

Consider $\Delta \in \mathbb{C}^{n \times n}$ such that $X=A+\Delta \in \mathcal{H}_{k}$. We know from Theorem 12 that this implies that $\lambda_{k+1}(S(A+\Delta)) \leq 0$ and that $\lambda_{n-k}(S(A+\Delta)) \geq 0$.

Consider $\tilde{\Delta}=Q^{*} S(\Delta) Q$ and partition it as follows

$$
Q^{*} S(A+\Delta) Q=\left[\begin{array}{ccc}
D_{1}+\tilde{\Delta}_{11} & \tilde{\Delta}_{12} & \tilde{\Delta}_{13} \\
\tilde{\Delta}_{21} & D_{2}+\tilde{\Delta}_{22} & \tilde{\Delta}_{23} \\
\tilde{\Delta}_{31} & \tilde{\Delta}_{32} & D_{3}+\tilde{\Delta}_{33}
\end{array}\right]
$$

where $D=\operatorname{diag}\left(D_{1}, D_{2}, D_{3}\right)$ is the diagonal of the eigendecomposition $S(A)=Q D Q^{*}$. In particular $D_{1} \in \mathbb{R}^{k_{+} \times k_{+}}$contains the eigenvalues of $S(A)$ which are strictly greater than 0 , and $D_{3} \in \mathbb{R}^{k_{-} \times k_{-}}$contains the eigenvalues of $S(A)$ strictly smaller than 0 .

- Assume that $k_{+}>k$. The matrix $Q^{*} S(A+\Delta) Q$ is Hermitian, hence by the interlacing inequalities we get

$$
\lambda_{k+1}\left(D_{1}+\tilde{\Delta}_{11}\right) \leq \lambda_{k+1}(S(A+\Delta)) \leq 0
$$

and then by Weyl's inequalities for each $k<i \leq k_{+}$

$$
\lambda_{i}=\lambda_{i}\left(D_{1}\right) \leq \lambda_{k+1}\left(D_{1}+\tilde{\Delta}_{11}\right)+\lambda_{i-k}\left(\tilde{\Delta}_{11}\right) \leq \lambda_{i-k}\left(\tilde{\Delta}_{11}\right),
$$

from which we obtain $\lambda_{i-k}\left(\tilde{\Delta}_{11}\right) \geq \lambda_{i}$, and hence

$$
\left\|\tilde{\Delta}_{11}\right\|_{F}^{2}=\sum_{i=1}^{k_{+}} \lambda_{i}\left(\tilde{\Delta}_{11}\right)^{2} \geq \sum_{i=k+1}^{k_{+}} \lambda_{i}^{2} .
$$

Note that (6) holds trivially also when $k_{+} \leq k$.

- Similarly, assume that $k_{-}>k$, and use again the interlacing inequalities to get

$$
\lambda_{k_{-}-k}\left(D_{3}+\tilde{\Delta}_{33}\right) \geq \lambda_{n-k}(S(A+\Delta)) \geq 0 .
$$

Using Weyl's inequalities for each $k<i \leq k_{-}$we can show that

$$
0 \leq \lambda_{k_{-}-k}\left(D_{3}+\tilde{\Delta}_{33}\right) \leq \lambda_{k_{-}+1-i}\left(D_{3}\right)+\lambda_{i-k}\left(\tilde{\Delta}_{33}\right)=\lambda_{n+1-i}+\lambda_{i-k}\left(\tilde{\Delta}_{33}\right),
$$

from which we obtain $\lambda_{i-k}\left(\tilde{\Delta}_{33}\right) \geq-\lambda_{n+1-i} \geq 0$, and hence

$$
\left\|\tilde{\Delta}_{33}\right\|_{F}^{2} \geq \sum_{i=k+1}^{k_{-}} \lambda_{i-k}\left(\tilde{\Delta}_{33}\right)^{2} \geq \sum_{i=k+1}^{k_{-}}\left(-\lambda_{n+1-i}\right)^{2}=\sum_{j=n+1-k_{-}}^{n-k} \lambda_{j}^{2} .
$$

We note that this equation holds trivially when $k_{-} \leq k$.

Combining the inequalities (6) and (7), and by Lemma 13 stating that $\|\Delta\|_{F}^{2} \geq\|S(\Delta)\|_{F}^{2}$, we get

$$
\|A-X\|_{F}^{2}=\|\Delta\|_{F}^{2} \geq\left\|\tilde{\Delta}_{11}\right\|_{F}^{2}+\left\|\tilde{\Delta}_{33}\right\|_{F}^{2} \geq \sum_{i=k+1}^{k_{+}} \lambda_{i}^{2}+\sum_{j=n+1-k_{-}}^{n-k} \lambda_{j}^{2},
$$

which is precisely $\|D-\hat{D}\|_{F}=\|A-\hat{A}\|_{F}$. This concludes the proof. 


\section{The Cayley transform}

Unitary and Hermitian structures are both special cases of normal matrices. Even more interestingly, it is known that they can be mapped one into the other through the use of the Cayley transform, defined as follows:

$$
\mathcal{C}(z):=\frac{z-i}{z+i}, \quad z \in \mathbb{C} \backslash\{-i\}
$$

The Cayley transform is a particular case of a Möbius transform, which permutes projective lines of the Riemann sphere. In particular, we have that $\mathcal{C}(\mathbb{R})=\mathbb{S}^{1}$. The inverse transform can be readily expressed as

$$
\mathcal{C}^{-1}(z)=i \cdot \frac{1+z}{1-z}, \quad z \in \mathbb{C} \backslash\{-1\}
$$

The fact that $\mathcal{C}(z)$ maps Hermitian matrices into unitary ones has been known for a long time [27,43. More recently, the observation that one can switch between low-rank perturbations of these structures has been exploited for develop fast algorithms for unitary-plus-low-rank and Hermitian-plus-low-rank matrices [6, 28,

Lemma 16. Let $A$ be an $n \times n$ matrix. Then we have the following.

- If $A$ does not have the eigenvalue $-i$ and $A$ is a rank $k$ perturbation of a Hermitian matrix, then $\mathcal{C}(A)$ will be a rank $k$ perturbation of a unitary matrix. Moreover, $\mathcal{C}(A)$ does not possess the eigenvalue 1.

- If $A$ does not have the eigenvalue 1 and is a rank $k$ perturbation of a unitary matrix, then $\mathcal{C}^{-1}(A)$ will be a rank $k$ perturbation of an Hermitian matrix, and $C^{-1}(A)$ does not possess eigenvalue $-i$.

Proof. We show that the Cayley transform (and its inverse) preserve the rank of the perturbation. Note that both $\mathcal{C}(z)$ and $\mathcal{C}^{-1}(z)$ are degree $(1,1)$ rational functions. For a rational function $r(z)$ of degree (at most) $(d, d)$ we know that, for any matrix $A$ and rank $k$ perturbation $E$, $r(A+E)-r(A)$ has rank at most $d k$. It remains to prove that perturbation stays exactly of rank $k$, and not less. Let $A$ be Hermitian plus rank (exactly) $k$, and by contradiction, assume we can write $\mathcal{C}(A)=Q+E$, with $Q$ unitary and $\operatorname{rank}(E)=k^{\prime}<k$. Then, we would have that $\mathcal{C}^{-1}(Q+E)=A$ is a Hermitian plus rank $k^{\prime \prime}$ matrix where $k^{\prime \prime} \leq k^{\prime}$, leading to a contradiction.

To prove that $\mathcal{C}(A)$ does not have 1 in the spectrum, it suffices to note that $\mathcal{C}(z)$ is a bijection of the Riemann sphere, and maps the point at $\infty$ to 1 . Since the eigenvalues of $\mathcal{C}(A)$ are $\mathcal{C}(\lambda)$, with $\lambda$ the eigenvalues of $A$, we see the eigenvalue 1 must be excluded. The same argument applies for the second case.

Creating this bridge between low-rank perturbations of unitary and Hermitian matrices enables to use the criterion that we have developed for detecting matrices in $\mathcal{H}_{k}$ to matrices in $\mathcal{U}_{k}$, and the opposite direction as well. In fact, in the next lemma, we show that we can obtain alternative proofs for the characterizations of $\mathcal{U}_{k}$ and $\mathcal{H}_{k}$ by simply applying the Cayley transform.

Lemma 17. The Cayley transformation implies that Theorem 3 and Theorem 12 are equivalent.

Proof. We start by proving that Theorem 12 implies Theorem 3 . Let $A$ be an arbitrary matrix, and assume that 1 is not an eigenvalue. We know by Lemma 16 that $A$ is in $\mathcal{U}_{k}$ if and only if 
$\mathcal{C}^{-1}(A) \in \mathcal{H}_{k}$. Now, $\mathcal{C}^{-1}(A)$ will be a rank $k$ perturbation of a Hermitian matrix if and only if the Hermitian matrix $\frac{1}{2 i}\left(\mathcal{C}^{-1}(A)-\mathcal{C}^{-1}(A)^{*}\right)$ has at most $k$ positve eigenvalues and $k$ negative ones. We can write

$$
\frac{1}{2 i}\left(\mathcal{C}^{-1}(A)-\mathcal{C}^{-1}(A)^{*}\right)=\frac{1}{2} \cdot\left[(A+I)^{-1}(A-I)+\left(A^{*}+I\right)^{-1}\left(A^{*}-I\right)\right],
$$

Let us do a congruence by left-multiplying by $(A+I)$ and right multiplying by $\left(A^{*}+I\right)$. This does not change the sign characteristic and yields

$$
\begin{aligned}
\frac{1}{2 i} \cdot(A+I)\left[\mathcal{C}^{-1}(A)-\mathcal{C}^{-1}(A)^{*}\right]\left(A^{*}+I\right) & =\frac{1}{2}\left[(A-I)\left(A^{*}+I\right)+(A+I)\left(A^{*}-I\right)\right] \\
& =A A^{*}-I .
\end{aligned}
$$

The matrix above has eigenvalues $\lambda_{i}:=\sigma_{i}^{2}(A)-1$, where $\sigma_{i}(A)$ are the singular values of $A$. Therefore, the positive eigenvalues of $\frac{1}{2 i}\left(\mathcal{C}^{-1}(A)-\mathcal{C}^{-1}(A)^{*}\right)$ correspond to singular values of $A$ larger than 1 , whereas the negative eigenvalues link to the singular values smaller than 1 . In particular, $A$ is unitary plus rank $k$, if and only if the characterization given in Theorem 3 is satisfied.

Let us now consider the case where $A$ has 1 as an eigenvalue. Then, we can multiply it by a unimodular scalar $\xi$ to get $A^{\prime}=\xi \cdot A$, where $A^{\prime}$ does not have 1 as an eigenvalue. Clearly $A^{\prime} \in \mathcal{U}_{k} \Longleftrightarrow A \in \mathcal{U}_{k}$, and $\sigma_{i}\left(A^{\prime}\right)=\sigma_{i}(A)$. Applying the previous steps to $A^{\prime}$ yields the characterization for $A$ as well, completing the proof.

The other implication (that is, Theorem 3 implies Theorem [12) can be obtained following the same steps backwards.

Remark 18. We emphasize that, although in principle the Cayley transform enables to study unitary matrices looking at Hermitian ones (and the other way around), it cannot be used to answer questions about the closest unitary or Hermitian matrix. In fact, this transformation does not preserve the distances.

\section{Reconstruction of the matrices}

Suppose we are given a matrix $A$, and we want to write it as a Hermitian-plus-low rank matrix 2 We present a strategy to recover the Hermitian and low-rank parts within $\mathcal{O}\left(n^{2} k\right)$ flops. We note that, if $A=H+G B^{*}$, with $H=H^{*}$, the Hermitian matrix $S(A)=\frac{1}{2 i}\left(A-A^{*}\right)$ has low-rank. Therefore, we can run a few steps of Lanczos to recover an approximation $S(A)=\frac{1}{2 i}\left(A-A^{*}\right) \approx$ $W T W^{*}$.

According to Theorem 12, we can use the eigenvalue decomposition of $S(A)$ to recover the best Hermitian-plus-low rank approximation, and the low-rank correction is described by Lemma 10 , Therefore, we construct this correction explicitly working with the matrix $T$, which we decompose as $T \approx \hat{B} \hat{C}^{*}+\hat{C} \hat{B}^{*}$, neglecting the eigenvalues smaller than a prescribed truncation threshold. This implies that we can write $\frac{1}{2 i}\left(A-A^{*}\right) \approx B C^{*}+C B^{*}$, where $C:=W \hat{C}, B:=W \hat{B}$. Then, we construct the final decomposition by setting $H=A-2 i C B^{*}$. The procedure is sketched in the pseudocode of Algorithm 1

Note that in the unlikely case the process terminates early $W T W^{*}$ is not equal to $S$, but only to its restriction on the maximal Krylov subspace. When failure is detected, we can restart the iterations with a randomly chosen $v$.

\footnotetext{
${ }^{2}$ In this section we will focus on Hermitian-plus-low-rank matrices; the idea in this section is also applicable to unitary-plus-low rank matrices.
} 


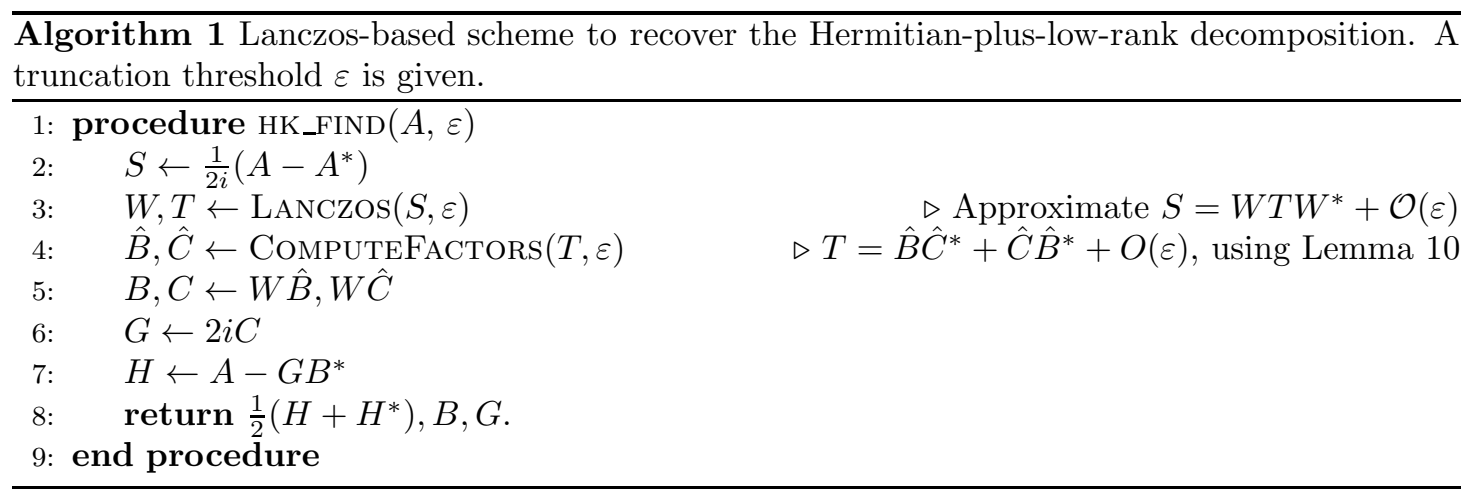

Remark 19. A reconstruction procedure can be obtained from any method to approximate the range of $S$ in $O\left(n^{2} k\right)$ flops; for instance, rank-revealing QR factorization [17, or randomized sampling [33]. Indeed, once one obtains an orthogonal basis $W$ for $\operatorname{Im} S$, one can compute $W^{*} S W=T$ and continue as above.

\section{$9 \quad$ Numerical experiments}

\subsection{Accuracy of the reconstruction procedure}

We have coded Algorithm 1 in Matlab, and ran some random tests to validate the procedure. The algorithm appears to be quite robust and succeeds, even in the case of noise, in retrieving a good decomposition.

In each test, we generated a random $n \times n$ Hermitian plus rank- $k$ matrix with the Matlab commands

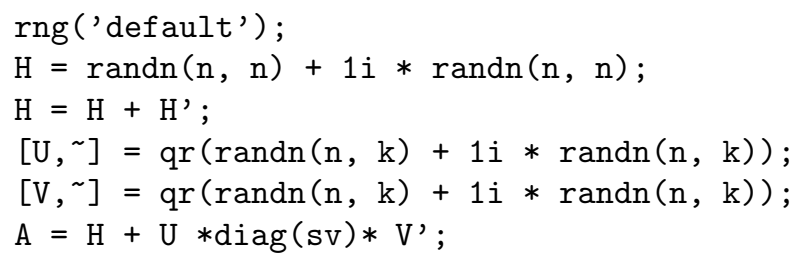

Here sv are logarithmically distributed singular values between 1 and a parameter $\sigma$.

We ran experiments with varying values of $n, k$, and $\sigma$; these results are in Figures 1, 2. and 3, respectively. We show in these tables the magnitude of the subdiagonal entries $\beta_{i}$ of the tridiagonal matrix $T$ produced in the Lanczos process. The graphs show that there is a sharp drop in their magnitude after $2 k$ steps, which is what is expected since $\operatorname{rank}(S)=2 k$, generically. Also, the magnitude of the intermediate values of $\beta_{i}$ reflects the decay in the singular values in the rank correction.

The legend also reports the relative residual in the reconstruction, computed as

$$
\operatorname{res}=\frac{\left\|\frac{1}{2}\left(H+H^{*}\right)+G B^{*}-A\right\|_{2}}{\|A\|_{2}}
$$

which is always below machine precision. 


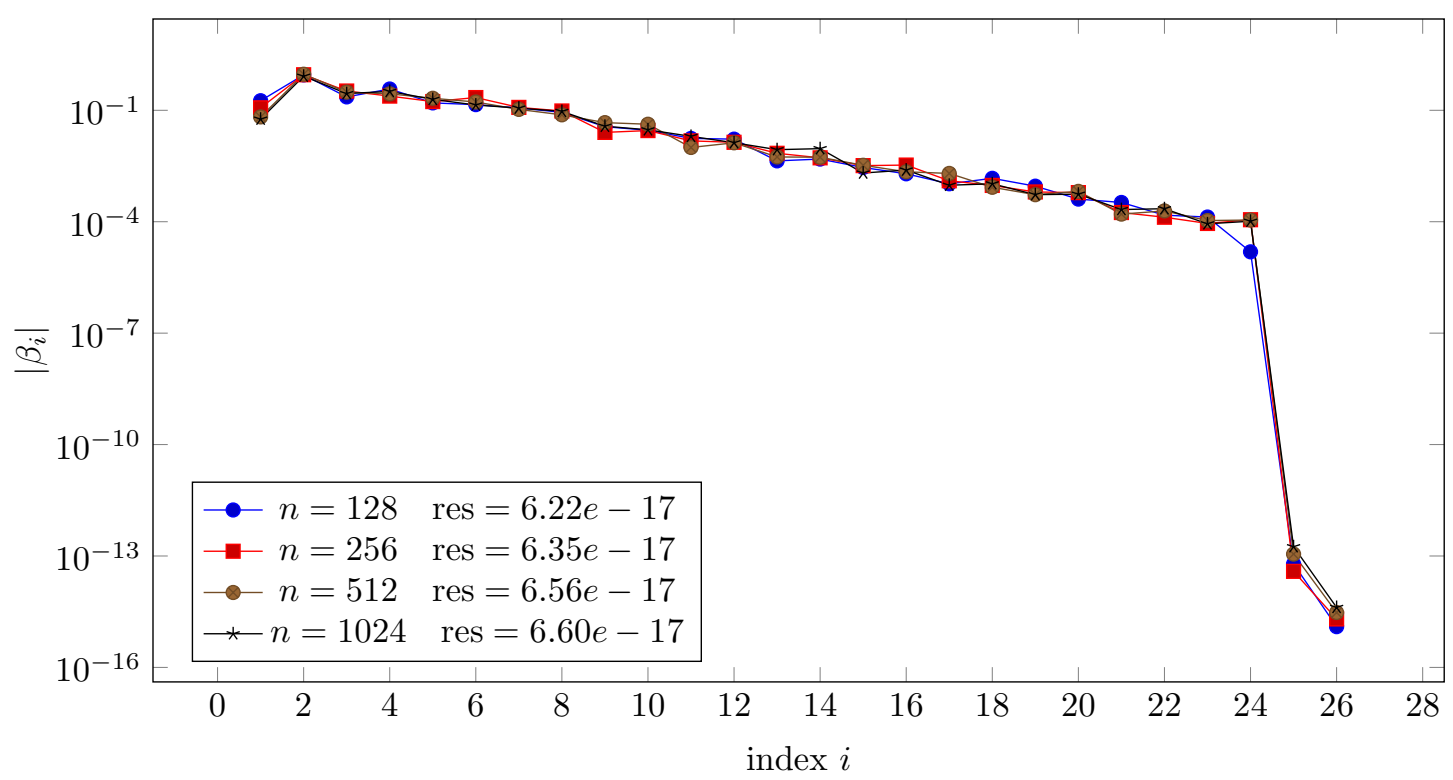

Figure 1: For $\sigma=1.0 e-4$ and $k=12$, magnitude of the subdiagonal entries of $T$ of size $n$.

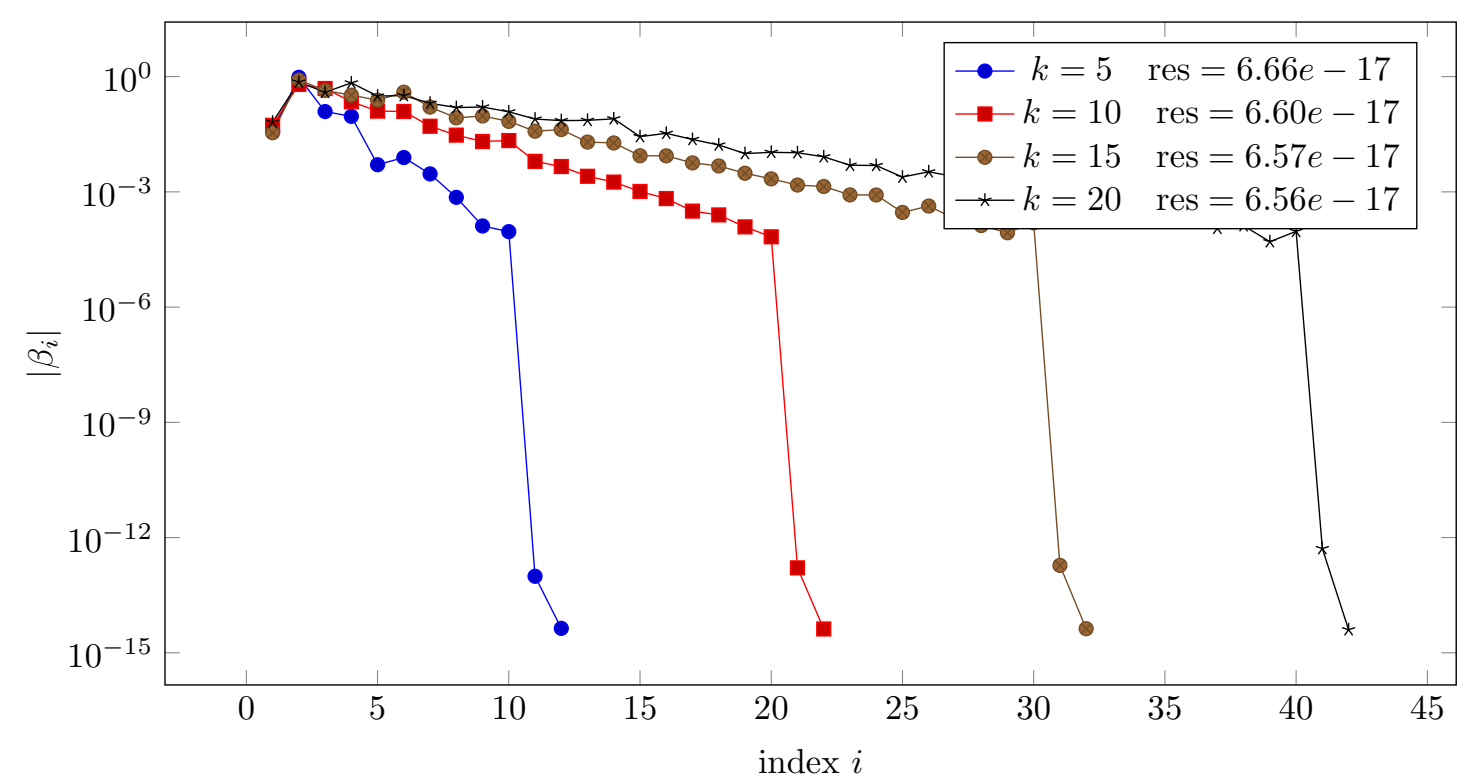

Figure 2: For $n=1024, \sigma=1.0 e-4$, magnitude of the subdiagonal entries of $T$ for various $k$. 


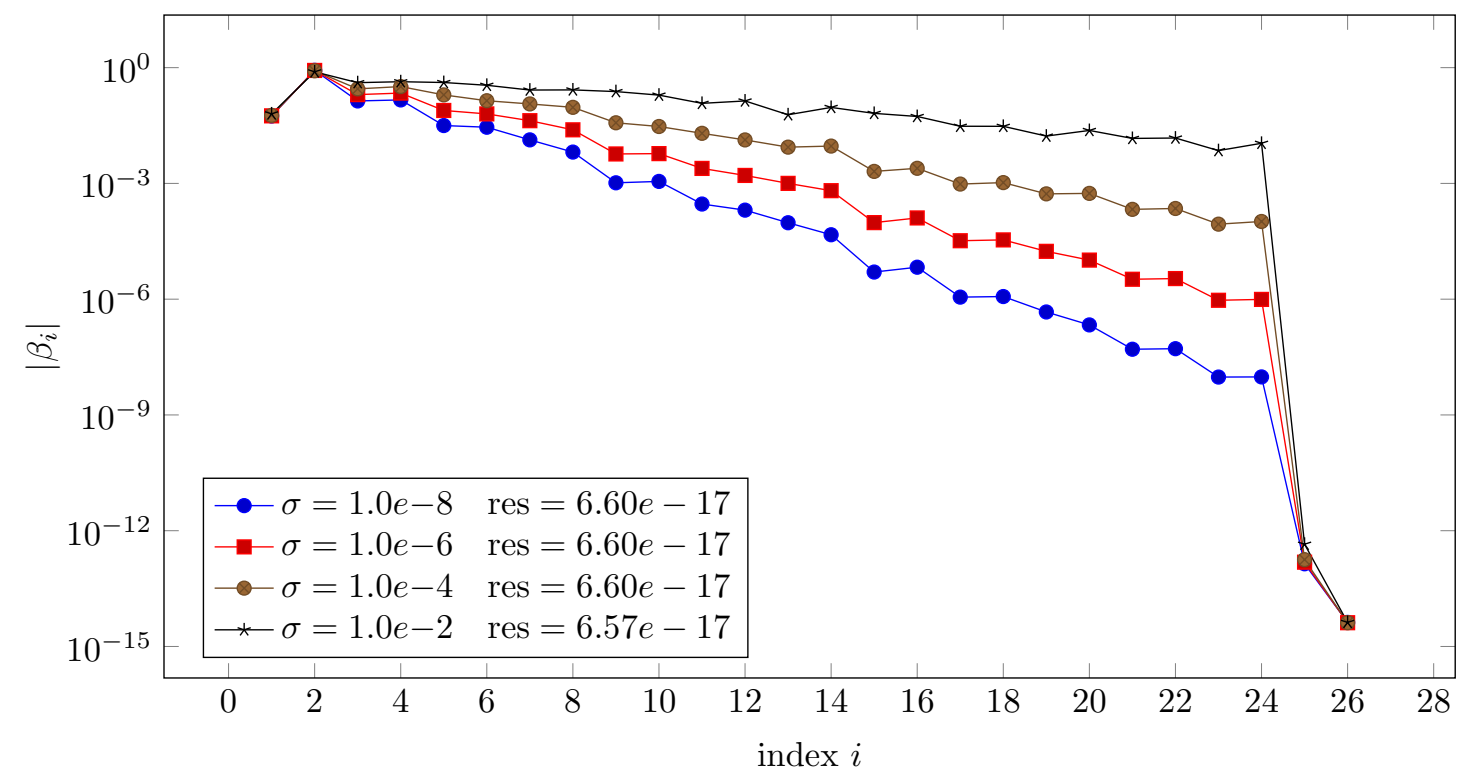

Figure 3: For $n=1024$ and $k=12$, magnitude of the subdiagonal entries of $T$ for different values of the smallest singular value.

\subsection{Structure loss in computing the Schur form}

One example of a natural question that can be answered using the tools developed in this paper is the following. Given a companion matrix $C$, in its Schur form $C=Q T Q^{*}$ the upper triangular factor $T$ is unitary-plus-rank-1, in exact arithmetic (since $C$ is so). As described in the introduction, several numerical methods in the literature try to exploit this structure, using special representations to enforce exactly the unitary plus rank 1 structure. If instead an approximation $\tilde{T}$ is computed using the standard QR algorithm (Matlab's schur(C)), can we measure the loss of structure in $\tilde{T}$, i.e., the distance between $\tilde{T}$ and the closest matrix which is unitary-plus-rank-1?

We have run some experiments in which this distance is computed using the formula in Theorem 7 in two different cases:

- The companion matrix of a polynomial whose roots are random numbers generated from a normal distribution with mean 0 and variance 1, i.e., Matlab's compan (poly (randn (n, 1)));

- The companion matrix of Wilkinson's polynomial, i.e., the polynomial with roots $1,2, \ldots, n$.

The singular values of $\tilde{T}$ have been computed using extended precision arithmetic, to help getting a more accurate result.

We display in Figure 4 the (relative) distance from structure

$$
\frac{\|\tilde{T}-X\|_{2}}{\|\tilde{T}\|_{2}}, \quad X=\arg \min _{X \in \mathcal{U}_{k}}\|\tilde{T}-X\|_{2}
$$

for several different matrix sizes $n$. This distance is always within a moderate multiple of the machine precision, which is to be expected because the Schur form is computed with a backward 


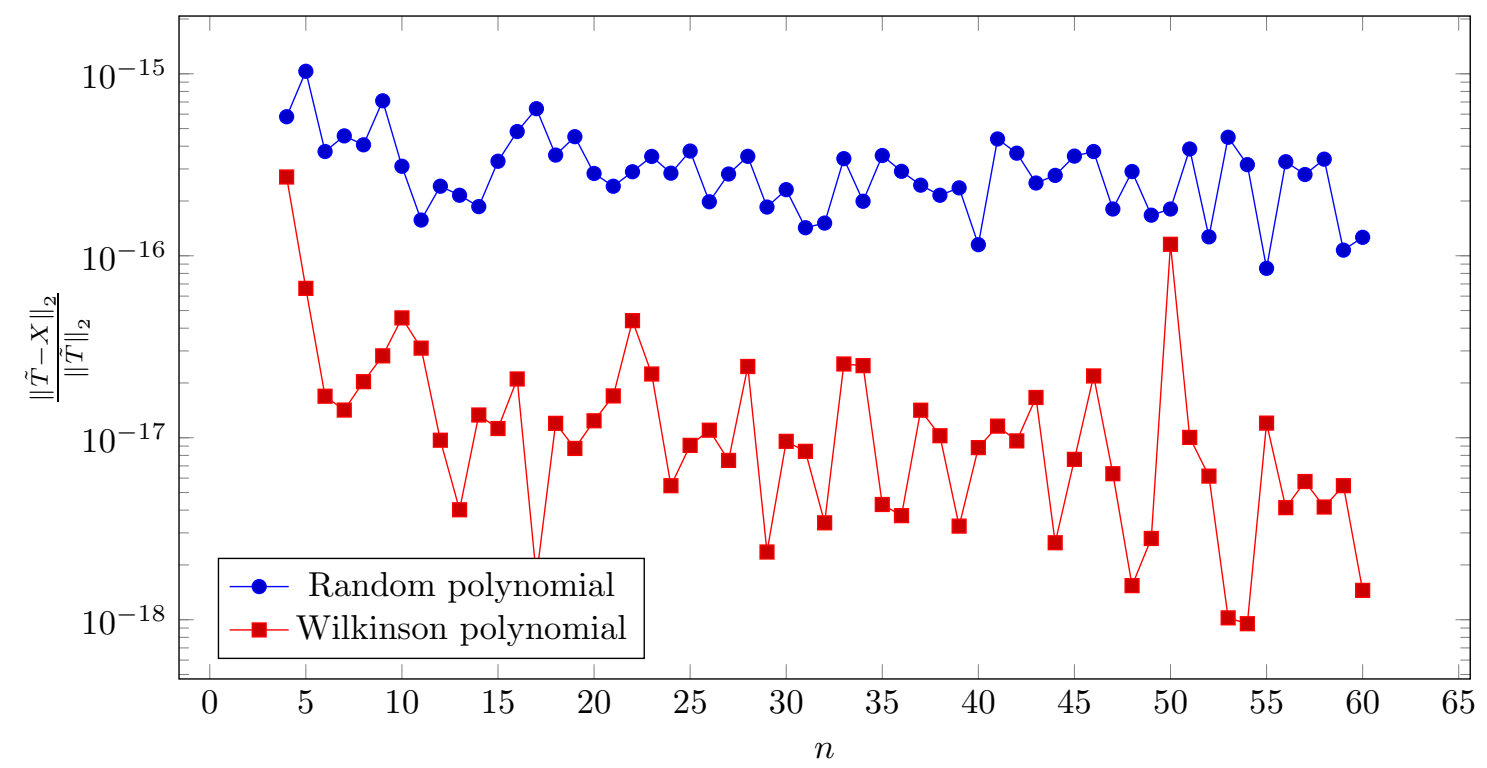

Figure 4: Relative distance from structure

stable algorithm. It appears that the loss of structure is less pronounced for the Wilkinson polynomial; note, though, that $\|T\|=\|C\|$ is much larger (for $n=60,\|C\| \approx 2 \times 10^{83}$ for the Wilkinson polynomial vs. $\|C\| \approx 3 \times 10^{7}$ for the random polynomial).

Another interesting quantity is

$$
\frac{\|\tilde{T}-X\|_{2}}{\|\tilde{T}-T\|_{2}}, \quad X=\arg \min _{X \in \mathcal{U}_{k}}\|\tilde{T}-X\|_{2},
$$

i.e., the relative amount (measured as a fraction in $[0,1]$ ) of the total error on $\tilde{T}$ that can be attributed to the loss of structure. We can approximate its denominator using the backward error $\|\tilde{T}-T\|_{2} \approx\left\|\tilde{Q} \tilde{T} \tilde{Q}^{*}-A\right\|_{2}$. If this ratio is close to 0 , then it means that the error introduced by the computation in the Schur form is almost tangent to the space $\mathcal{U}_{k}$, while if it is close to 1 the error is almost perpendicular to $\mathcal{U}_{k}$. We display this quantity in Figure 5 , It is again smaller for the Wilkinson polynomial, and in both cases it seems to decrease slowly as the dimension $n$ increases.

\section{Conclusions}

We have provided explicit conditions under which a matrix is unitary (resp. Hermitian) plus low rank, and have given a construction for the closest unitary (resp. Hermitian) plus rank $k$ to a given matrix $A$, in both the spectral and the Frobenius norm.

We have presented an algorithm based on the Lanczos iteration to construct explicitly, given a matrix $A \in \mathcal{H}_{k}$ (where $k$ is not known a priori), a representation of the form $A=H+G B^{*}$, where $H$ is Hermitian and $G B^{*}$ is a rank- $k$ correction. A variant for unitary-plus-low-rank matrices can be obtained in a similar fashion. 


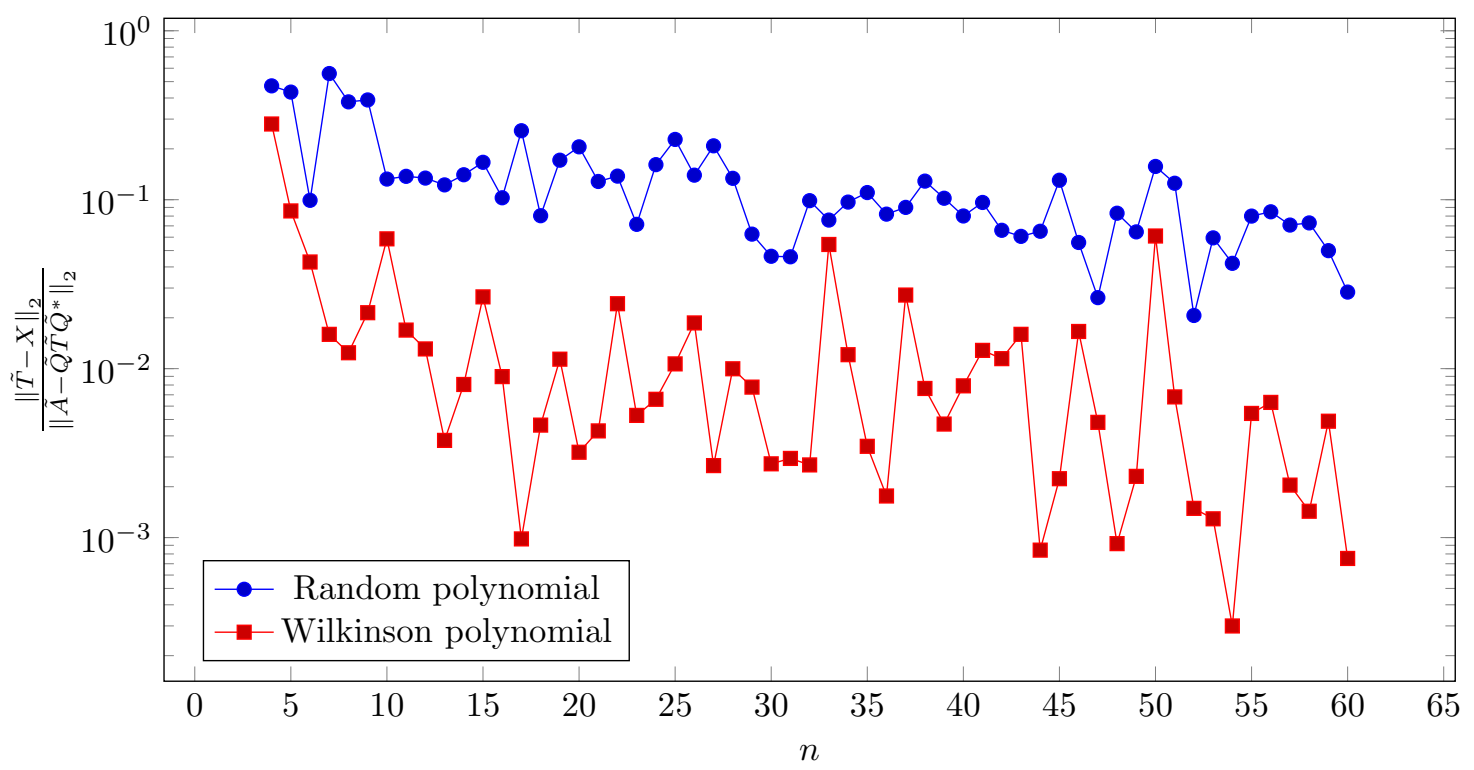

Figure 5: Percentage of error due to structure loss

An example of an application that is enabled by the theory developed in this paper is presented, i.e., assessing the loss of structure in the (unstructured) QR iteration.

\section{References}

[1] EISCOR eigenvalue solvers based on unitary core transformations. https://github.com/eiscor/eiscor.

[2] LAPACK linear algebra package. https://www.netlib.org/lapack/.

[3] J. L. Aurentz, T. Mach, L. Robol, R. Vandebril, and D. S. Watkins. Core-Chasing Algorithms for the Eigenvalue Problem. Number 13 in Fundamentals of Algorithms. SIAM, 2018.

[4] J. L. Aurentz, T. Mach, L. Robol, R. Vandebril, and D. S. Watkins. Fast and backward stable computation of the eigenvalues of matrix polynomials. Mathematics of Computation, 88:313-347, 2019.

[5] J. L. Aurentz, T. Mach, R. Vandebril, and D. S. Watkins. Fast and stable unitary QR algorithm. Electronic Transactions on Numerical Analysis, 44:327-341, November 2015.

[6] J. L. Aurentz, T. Mach, R. Vandebril, and D. S. Watkins. Computing the eigenvalues of symmetric tridiagonal matrices via a Cayley transformation. Electronic Transactions on Numerical Analysis, 46:447-459, 2017.

[7] R. Bevilacqua, G. M. Del Corso, and L. Gemignani. A CMV-based eigensolver for companion matrices. SIAM Journal on Matrix Analysis and Applications, 36(3):1046-1068, 2015. 
[8] R. Bevilacqua, G. M. Del Corso, and L. Gemignani. Fast QR iterations for unitary plus low rank matrices. ArXiv e-prints, Oct. 2018.

[9] D. A. Bini, P. Boito, Y. Eidelman, L. Gemignani, and I. Gohberg. A fast implicit QR eigenvalue algorithm for companion matrices. Linear Algebra and its Applications, 432(8):20062031, 2010.

[10] D. A. Bini, Y. Eidelman, L. Gemignani, and I. Gohberg. Fast QR eigenvalue algorithms for Hessenberg matrices which are rank-one perturbations of unitary matrices. SIAM Journal on Matrix Analysis and Applications, 29(2):566-585, 2007.

[11] D. A. Bini and L. Robol. On a class of matrix pencils and $\ell$-ifications equivalent to a given matrix polynomial. Linear Algebra and its Applications, 502:275-298, 2016.

[12] D. A. Bini and L. Robol. Quasiseparable Hessenberg reduction of real diagonal plus low rank matrices and applications. Linear Algebra and its Applications, 502:186-213, 2016.

[13] P. Boito, Y. Eidelman, and L. Gemignani. Implicit QR for rank-structured matrix pencils. BIT, 54:85-111, 2013.

[14] P. Boito, Y. Eidelman, L. Gemignani, and I. Gohberg. Implicit QR with compression. Indagationes Mathematicae, 23(4):733-761, 2012.

[15] A. Bunse-Gerstner and L. Elsner. Schur parameter pencils for the solution of the unitary eigenproblem. Linear Algebra and its Applications, 154-156:741-778, 1991.

[16] M. J. Cantero, L. Moral, and L. Velázquez. Five-diagonal matrices and zeros of orthogonal polynomials on the unit circle. Linear Algebra and its Applications, 362:29-56, 2003.

[17] T. F. Chan. Rank revealing $Q R$ factorizations. Linear Algebra and its Applications, 88/89:67-82, 1987.

[18] S. Chandrasekaran and M. Gu. Fast and stable eigendecomposition of symmetric banded plus semi-separable matrices. Linear Algebra and its Applications, 313:107-114, 2000.

[19] S. Chandrasekaran, M. Gu, J. Xia, and J. Zhu. A fast QR algorithm for companion matrices. Operator Theory: Advances and Applications, 179:111-143, 2007.

[20] F. De Terán, F. M. Dopico, and J. Pérez. Condition numbers for inversion of fiedler companion matrices. Linear Algebra Appl., 439(4):944-981, 2013.

[21] G. M. Del Corso, F. Poloni, L. Robol, and R. Vandebril. Factoring block Fiedler Companion Matrices. In Proceedings of the Structured Matrices in Numerical Linear Algebra: Analysis, Algorithms and Applications conference in Cortono, Italy, 201\%. Springer, to appear.

[22] S. Delvaux. Rank Structured Matrices. PhD thesis, Department of Computer Science, Katholieke Universiteit Leuven, Celestijnenlaan 200A, 3000 Leuven (Heverlee), Belgium, June 2007.

[23] J. W. Demmel, O. A. Marques, B. N. Parlett, and C. Vömel. Performance and accuracy of LAPACK's symmetric tridiagonal eigensolvers. SIAM Journal on Scientific Computing, 30(3):1508-1526, 2008.

[24] I. S. Dhillon, B. N. Parlett, and C. Vömel. The design and implementation of the MRRR algorithm. ACM Transactions on Mathematical Software, 32(4):533-560, Dec. 2006. 
[25] Y. Eidelman, L. Gemignani, and I. C. Gohberg. Efficient eigenvalue computation for quasiseparable Hermitian matrices under low rank perturbation. Numerical Algorithms, 47(3):253-273, March 2008.

[26] L. Elsner and K. D. Ikramov. Normal matrices: An update. Linear Algebra and its Applications, 285:291-303, 1998.

[27] J. N. Franklin. Matrix Theory. Dover publications inc., Mineola, New York, USA, 2012.

[28] L. Gemignani. A unitary Hessenberg QR-based algorithm via semiseparable matrices. Journal of Computational and Applied Mathematics, 184:505-517, 2005.

[29] L. Gemignani and L. Robol. Fast Hessenberg reduction of some rank structured matrices. SIAM Journal on Matrix Analysis and Applications, 38(2):574-598, 2017.

[30] W. B. Gragg. The QR algorithm for unitary Hessenberg matrices. Journal of Computational and Applied Mathematics, 16:1-8, 1986.

[31] W. B. Gragg and L. Reichel. A divide and conquer method for unitary and orthogonal eigenproblems. Numerische Mathematik, 57:695-718, 1990.

[32] R. Grone, C. R. Johnson, E. M. Sa, and H. Wolkowicz. Normal matrices. Linear Algebra and its Applications, 87:213-225, 1987.

[33] N. Halko, P. G. Martinsson, and J. A. Tropp. Finding structure with randomness: probabilistic algorithms for constructing approximate matrix decompositions. SIAM Review, 53(2):217-288, 2011.

[34] R. A. Horn and C. R. Johnson. Matrix Analysis. Cambridge University Press, Cambridge, second edition, 2013.

[35] B. N. Parlett. The Symmetric Eigenvalue Problem, volume 20 of Classics in Applied Mathematics. SIAM, Philadelphia, Pennsylvania, USA, 1998.

[36] L. Robol. Exploiting rank structures for the numerical treatment of matrix polynomials. PhD thesis, Scuola Normale Superiore di Pisa, 2015.

[37] L. Robol and R. Vandebril. Efficient Ehrlich-Aberth iteration for finding intersections of interpolating polynomials and rational functions. Linear Algebra and its Applications, 542:282-309, 2018.

[38] L. Robol, R. Vandebril, and P. V. Dooren. A framework for structured linearizations of matrix polynomials in various bases. SIAM Journal on Matrix Analysis and Applications, 38(1):188-216, 2017.

[39] R. C. Thompson. Principal submatrices. IX. Interlacing inequalities for singular values of submatrices. Linear Algebra and its Applications, 5:1-12, 1972.

[40] M. Van Barel, R. Vandebril, P. Van Dooren, and K. Frederix. Implicit double shift $Q R$ algorithm for companion matrices. Numerische Mathematik, 116(2):177-212, 2010.

[41] R. Vandebril and G. M. Del Corso. An implicit multishift $Q R$-algorithm for Hermitian plus low rank matrices. SIAM Journal on Scientific Computing, 32(4):2190-2212, 2010.

[42] R. Vandebril, M. Van Barel, and N. Mastronardi. Matrix Computations and Semiseparable Matrices: Linear Systems, volume 1. JHU Press, 2007. 
[43] J. von Neumann. Allgemeine Eigenwerttheorie Hermitescher Funktionaloperatoren. Mathematische Annalen, 102:49-131, 1930.

[44] D. S. Watkins. The Matrix Eigenvalue Problem: GR and Krylov Subspace Methods. SIAM, Philadelphia, USA, 2007. 